\title{
Simulação Multiagente para o Apoio às Políticas de Desarmamento
}

\author{
Daniel P. Ferreira ${ }^{1}$, Diana F. Adamatti ${ }^{1}$, Tatiane R. Bastos ${ }^{1}$ \\ ${ }^{1}$ Programa de Pós-Graduação em Modelagem Computacional (PPGMC) - \\ Universidade Federal do Rio Grande (FURG) - Rio Grande - RS - Brasil \\ \{daniielferr, dianaadamatti, tatianebastos\} afurg.br
}

\begin{abstract}
The objective of this study is to verify the efficiency of firearms, rifle and pistol, in events of mass shootings in the closed environment and which parameters influence the number of victims, in order to support the analysis and elaboration of public policies. To do this, we developed a Multi-Agent Simulation using the NetLogo software. The results show that the type of weapon used in the event has a great influence on the number of victims. In addition, it is also possible that the difference in the number of victims of the two weapons will decrease as the amount of ammunition increases and the size of the exit door decreases, due to the funnel effect.
\end{abstract}

Resumo. O objetivo da presente pesquisa consiste em verificar a eficácia das armas de fogo, fuzil e pistola, em eventos de tiroteios em massa em ambiente fechado e quais os parâmetros que influenciam no número de vítimas, a fim de apoiar a análise e elaboração de políticas públicas. Para isso, realizou-se a Simulação Multiagente através do software NetLogo. Os resultados mostram que o tipo de arma utilizada no evento tem grande influência no número de vítimas. Além disso, também observou-se que a diferença no número de vítimas das duas armas diminui à medida em que se aumenta a quantidade de munição e se diminui a dimensão da porta de saída, devido ao efeito funil.

\section{Introdução}

As armas de fogo são importantes instrumentos motivadores da violência urbana. Segundo a Organização das Nações Unidas [ONU 2018], o controle de armas e as medidas de desarmamento podem auxiliar à segurança nacional e humana no século XXI e deve ser parte integrante do sistema de segurança coletiva, direcionando-se os esforços não apenas ao controle de armas nucleares, mas também para impedir e reverter o excesso de acúmulo de todos os outros tipos de armas.

Neste contexto, a discussão sobre políticas de restrição ao acesso de civis a armas de fogo foi levantada em diversos países [Luca et al 2020; Chapman et al 2006; Lemieux 2014; Ferro e Teixeira 2019; McPhedran e Baker 2012]. O debate sobre o controle de armas repercute principalmente mediante eventos de tiroteios em massa, que segundo Luca et al (2020), podem criar "janelas políticas" durante as quais a legislatura torna-se aberta ao diálogo.

Embora os tiroteios em massa sejam eventos pouco recorrentes, causam um grande impacto na sociedade, pois além de ocorrerem rapidamente e não poderem ser previstos, acontecem em lugares onde as vítimas se sentem seguras, como escolas, campi universitários, cinemas, teatros, shopping centers e espaços públicos [Briggs e Kennedy 2016]. 
No Brasil, mesmo com o Estatuto de Desarmamento em vigor desde 2002, ao menos oito escolas foram palco de ataques em massa com uso de armas de fogo, resultando em 28 mortos e 41 feridos, e em metade dos casos, os atiradores utilizaram armas que estavam armazenadas em suas casas [Folha de São Paulo, 2019].

Os Sistemas Multiagente (SMA) estudam o comportamento de um conjunto independente de agentes com diferentes características, evoluindo em um ambiente comum. Esses agentes interagem uns com os outros, e tentam executar suas tarefas de forma cooperativa, compartilhando informações, evitando conflitos e coordenando a execução das atividades. Adicionalmente, o uso de simulação como ferramenta de apoio a tomada de decisão é bastante eficiente, porque é possível verificar detalhes com grande precisão.

Desta forma, o presente estudo tem como objetivo verificar quais aspectos ou parâmetros influenciam no número de vítimas em casos de tiroteios em massa, a fim de contribuir com a análise e o planejamento de políticas públicas que tratem da liberação de armas de fogo e segurança pública. Para isso, foi realizada a modelagem baseada em agente através do software NetLogo.

Além desta seção introdutória, é apresentado o referencial teórico na seção 2, o modelo proposto é explicitado na seção 3, seguida dos resultados e considerações finais, tratados nas seções 4 e 5, respectivamente, e por fim, são apresentadas as referências.

\section{Trabalhos correlatos}

A Simulação Baseada em Agentes tem sido utilizada em pesquisas que visam modelar situações de combate, pois permite examinar computacionalmente as diversas ações dos agentes nos diferentes cenários.

Hayes e Hayes (2014) criaram uma simulação baseada em agentes para examinar os principais parâmetros em tiroteios em massa, com o objetivo de verificar se a proposta de lei da Senadora Dianne Feinstein, da Califórnia (EUA), para limitar o acesso às armas de assalto seria eficaz na redução do número de vítimas. Através das simulações, os autores concluíram que a proposta de lei não influenciaria na diminuição do número de mortes, já que o único parâmetro que reduziu o número de vítimas foi a taxa de tiros, o que não está contemplado na proposta de lei, que não engloba espingardas, rifles e pistolas semiautomáticas.

Já o trabalho de Briggs e Kennedy (2016) utiliza a modelagem baseada em agentes para explorar o potencial de reduzir o número de mortes caso um grupo de vítimas desarmadas enfrentem ostensivamente o atirador, como ocorreu em um ataque a trem de Amsterdã para Paris, em 2015. Os resultados mostraram que a resistência desarmada a um atirador ativo pode reduzir substancialmente o número de vítimas, no entanto, o risco de morte dos combatentes aumenta, principalmente caso o atirador não seja subjugado. Verificaram também que quanto maior a proporção de combatentes na população, maior a probabilidade do atirador ser subjugado.

No trabalho de Xi e Chan (2019), um modelo baseado em agente combinado a um sistema de informações georreferenciadas é utilizado para simular ataques com facas e ataques com armas em um campus de universidade chinesa. A pesquisa teve como motivação o maior número de ataques com facas na China, possivelmente devido à proibição de armas de fogo. Os autores examinaram computacionalmente as consequências das ações das vítimas durante o ataque, comparando os cenários de ataques 
a faca e de ataques com armas de fogo. Os resultados mostraram que as pessoas devem unirem-se para subjugar o invasor no caso de ataque a faca, enquanto nos casos de ataque com arma de fogo, o indicado é esconderem-se e aguardar as autoridades policiais, caso não possam subjugar imediatamente o agressor.

Diante o exposto, percebe-se que a utilização de agentes para a análise dos diversos cenários de ataques com armas de fogo em massa, a fim de verificar quais fatores influenciam no número de vítimas, consiste em uma importante ferramenta para o entendimento dos eventos, sendo portanto efetiva para o apoio aos gestores públicos na elaboração de políticas públicas.

\section{Modelo Proposto}

Nesta seção, apresenta-se inicialmente o modelo base proposto por Hayes e Hayes (2014). A retomada desse trabalho se dá em virtude de ter ele servido como base para a construção do modelo proposto na presente pesquisa. Na sequência, é apresentada a construção do modelo, que considera dois tipos de armas de fogo, fuzil e pistola.

\subsection{Modelo de Hayes e Hayes (2014)}

Hayes e Hayes (2014) realizaram uma modelagem baseada em agentes, a fim de analisar a eficácia do projeto de lei da senadora da Califórnia (EUA), Dianne Feinstein, que visava a proibição da venda de fuzis de assalto a civis. Este projeto teve o objetivo de diminuir o número de mortos e feridos em tiroteios em massa, tornando ilegal a compra de fuzis de assalto e também armas com carregadores de alta capacidade ou com características militares.

Com a modelagem baseada em agentes, verificaram a interação entre indivíduos em um cenário pré-definido, onde um agente (atirador) abre fogo contra os outros agentes (civis e seguranças). Cada agente tem um papel definido e age de acordo com este. A simulação é considerada completa quando todos os civis foram baleados ou escaparam, ou se o atirador for baleado ou atacado.

eles:

Alguns pontos em relação ao cenário da simulação devem ser destacados, são

- O atirador conta com o elemento surpresa, ou seja, ele sempre conseguirá atirar primeiro que os seguranças;

- São utilizados no cálculo da simulação: velocidade de corrida dos agentes, distância até a saída, alcance da arma, capacidade da arma, número de balas disparadas e desvio padrão (próprio da arma); e

- Os civis e seguranças são escolhidos aleatoriamente.

O número de pessoas atingidas no modelo de Hayes e Hayes é dado por uma equação comum de mínimos quadrados (Equação 1).

$$
n=-4,4 s+0,004 a+0,06 c+0,4 t+0,4 p+8,6
$$

Onde:

n é o número de pessoas baleadas;

s é o número de guardas de segurança; 
a é o alcance da arma;

c é a capacidade do carregador;

t é a taxa de tiro; e

p é a precisão da mira.

Assim que a simulação inicia e o atirador abre fogo, os civis começam a se dirigir para a porta de saída do local provocando uma aglomeração no local de evasão (efeito funil), aumentando assim a probabilidade de acerto do atirador. O modelo utiliza mínimos quadrados para definir os parâmetros que afetam o número de pessoas atingidas.

Por fim a simulação conclui a ineficácia da proibição proposta pela senadora, uma vez que em seu conteúdo não há restrições na taxa de tiro, nem na utilização de carregadores destacáveis, o que manteria permitido pistolas semiautomáticas, espingardas e rifles. Visto que à medida em que as pessoas se aproximam da saída, o atirador aumenta sua área de acerto, a proibição de fuzis de assalto não iria interferir no número de pessoas baleadas.

Alguns fatores podem realmente contribuir na diminuição do número de vítimas, como o aumento do número de seguranças no local, menor taxa de tiro da arma e menor quantidade de balas nos carregadores destacáveis.

\subsection{Modelo Proposto}

$\mathrm{Na}$ presente pesquisa, buscou-se identificar os fatores que interferem no número de vítimas em tiroteios em massa, através de simulação multiagente utilizando o software NetLogo. O modelo permite simular a interação entre indivíduos em um cenário fechado pré-definido, onde um agente (atirador) abre fogo contra os outros agentes (população). Foi considerado ambiente fechado um cenário delimitado por paredes e com uma porta de saída, no qual os agentes presentes no ambiente interno podem evacuar através da porta de saída. Cada agente na simulação tem um papel definido e irá agir de acordo com este, sendo a simulação considerada completa quando todos os civis forem baleados ou se o atirador for neutralizado.

O cenário consiste em um ambiente fechado de tamanho padrão correspondente a $3.025 \mathrm{~m}^{2}$. Foram considerados dois agentes, o atirador e os civis ou população. $\mathrm{O}$ atirador fica localizado no centro do cenário. A população inicial varia de 100 a 1.000 e movimenta-se randomicamente, dirigindo-se em direção a porta assim que o atirador abre fogo. Os agentes civis são homogéneos, logo possuem o mesmo comportamento e dirigem-se todos em direção a saída até evacuarem todo o ambiente ou o atirador ficar sem munição, quando o mesmo é considerado neutralizado e a simulação é findada.

É possível selecionar o tipo de arma a ser simulada, fuzil ou pistola. O Fuzil utilizado na simulação é o modelo Colt M4, que faz 30 disparos em 2,5 segundos por carregador, sendo gastos 7,5 segundos para a troca do carregador. Já a pistola utilizada é Sig Sauer P320, que faz 15 disparos em 5 segundos por carregador, sendo necessário mais 5 segundos para a troca do carregador. A munição que o atirador dispõe e que pode ser portada por ele, não apenas na arma, varia de 15 a 500 .

Considerando-se o efeito funil observado na pesquisa de [Hayes e Hayes 2014], que consiste na aglomeração de pessoas que se forma na porta de saída ao tentarem evadir do local, na presente simulação objetiva-se verificar também o efeito do tamanho da porta 
de saída no número de vítimas. Desta forma, é possível realizar a simulação alterando o tamanho da porta de 1 a 5 . A Figura 1 apresenta a interface da simulação.

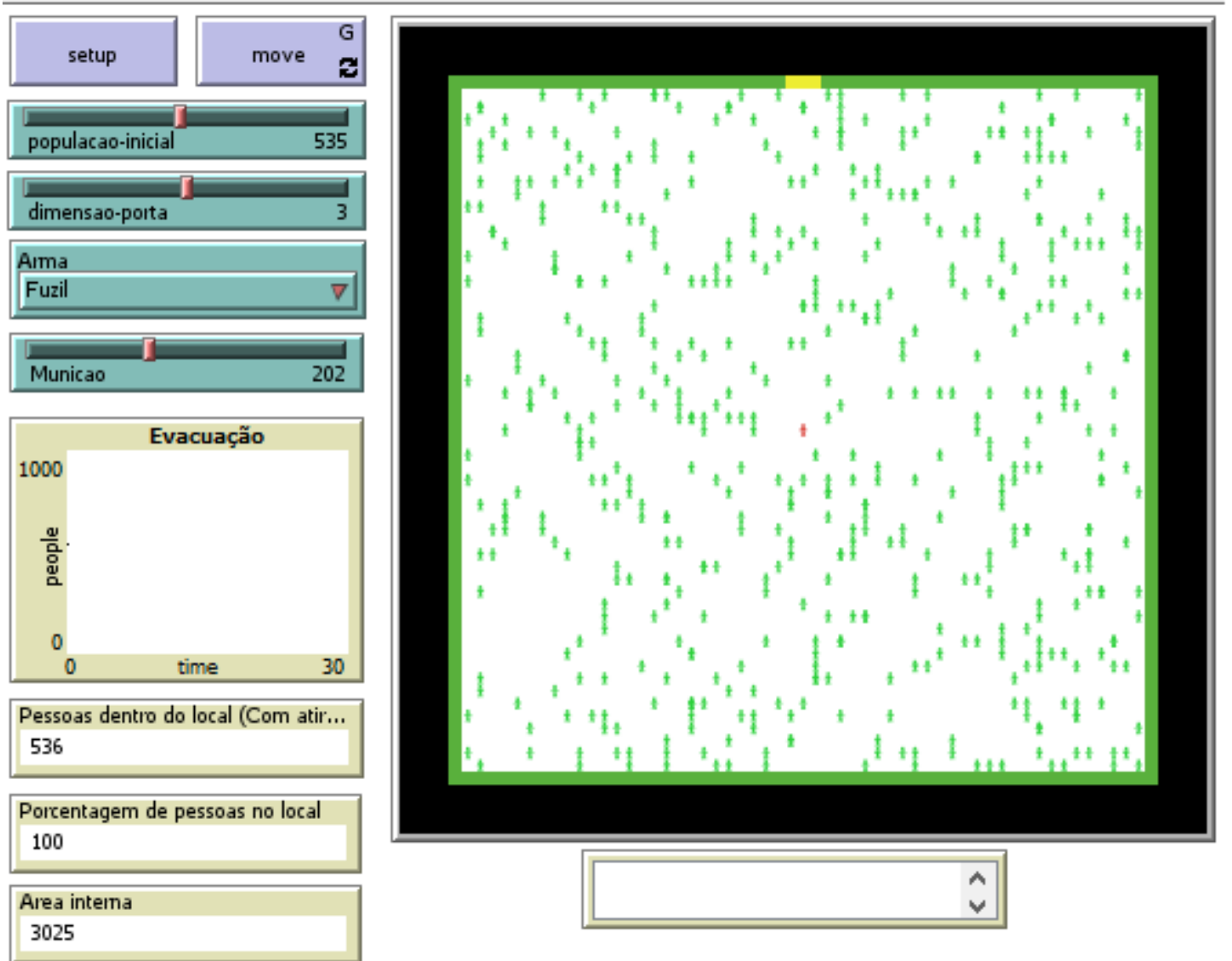

Figura 1. Interface do modelo proposto

Selecionando-se o botão setup, o contador é zerado e os parâmetros iniciais são reestabelecidos. Ao selecionar go, a simulação inicia com o atirador efetuando o primeiro disparo e as pessoas se movimentam aleatoriamente em direção à saída. A simulação é concluída quando todas as pessoas conseguem escapar, ficando na parte interna do cenário apenas o atirador e as vítimas (evacuação completa) ou quando acaba a munição do atirador (atirador neutralizado).

\section{Resultados}

Foram realizadas seis simulações com parâmetros de entrada diferentes, sendo estes escolhidos de forma randômica dentro dos valores mínimos e máximos definidos na programação do modelo, onde: número máximo de pessoas no ambiente entre 100 e 1000 , dimensão da porta entre 1 e 5, munição das armas (fuzil e pistola) entre 15 e 500. Para cada simulação foram realizados três testes. Na primeira simulação, foi definida a população inicial de $\mathbf{5 4 0}$ pessoas, dimensão da porta igual a $\mathbf{2}$ e a arma escolhida foi a pistola com munição igual a 130. Na segunda simulação, a população inicial foi de $\mathbf{5 4 0}$ pessoas, dimensão da porta igual a $\mathbf{2}$ e a arma escolhida foi o fuzil com munição igual a 130. Na Tabela 1 são apresentados os resultados das duas primeiras simulações. 
Tabela 1. Resultados das Simulações 1 e 2

\begin{tabular}{|l|c|c|c|c|}
\hline \multicolumn{5}{|c|}{ Simulação 1 } \\
\hline \multicolumn{1}{|c|}{ Parâmetros de análise } & Teste 1 & Teste 2 & Teste 3 & Média \\
\hline Pessoas no local & 63 & 69 & 60 & 64 \\
\hline Pessoas no local (\%) & 11,48 & 12,59 & 10,93 & 11,67 \\
\hline Vítimas & 62 & 68 & 59 & 63 \\
\hline Disparos & 76 & 102 & 95 & 91 \\
\hline Pessoas não atingidas no local & 0 & 0 & 0 & 0 \\
\hline Saída & $\begin{array}{c}\text { Evacuação } \\
\text { completa }\end{array}$ & $\begin{array}{c}\text { Evacuação } \\
\text { completa }\end{array}$ & $\begin{array}{c}\text { Evacuação } \\
\text { completa }\end{array}$ & \\
\hline \multicolumn{1}{|c|}{ Simulação 2 } & & \\
\hline Parâmetros de análise & Teste 1 & Teste 2 & Teste 3 & Média \\
\hline Pessoas no local & 428 & 401 & 389 & 406 \\
\hline Pessoas no local (\%) & 79,07 & 74,07 & 71,85 & 75,00 \\
\hline Vítimas & 117 & 108 & 114 & 113 \\
\hline Disparos & 130 & 130 & 130 & 130 \\
\hline Pessoas não atingidas no local & 310 & 292 & 274 & 292 \\
\hline Saída & $\begin{array}{c}\text { Atirador } \\
\text { neutralizado }\end{array}$ & $\begin{array}{c}\text { Atirador } \\
\text { neutralizado }\end{array}$ & $\begin{array}{c}\text { Atirador } \\
\text { neutralizado }\end{array}$ & \\
\hline
\end{tabular}

Observa-se que a simulação realizada com a pistola foi finalizada quando a evacuação completa ocorreu, enquanto na simulação realizada com fuzil o atirador foi neutralizado, ou seja, o número de vítimas apenas não foi maior, pois a munição do atirador acabou. A simulação realizada com o fuzil resultou em média 50 vítimas a mais do que a realizada com a pistola, o que mostra que o tipo de arma tem grande efeito sobre o número de mortes em um evento de tiroteio em massa.

Na terceira simulação, os parâmetros de entrada foram população inicial de $\mathbf{6 5 0}$ pessoas, dimensão da porta igual a $\mathbf{3}$ e a arma utilizada foi a pistola com munição igual a 250. Já a simulação 4 teve como parâmetros os mesmos montantes para a população inicial e munição e a mesma dimensão de porta, porém a arma utilizada foi o fuzil. A Tabela 2 apresenta os resultados destas duas simulações.

Tabela 2. Resultados das Simulações 3 e 4

\begin{tabular}{|l|c|c|c|c|}
\hline \multicolumn{7}{|c|}{ Simulação 3 } \\
\hline Parâmetros de análise & Teste 1 & Teste 2 & Teste 3 & Média \\
\hline Pessoas no local & 60 & 65 & 82 & 69 \\
\hline Pessoas no local (\%) & 9,08 & 9,85 & 12,46 & 10,46 \\
\hline Vítimas & 59 & 64 & 81 & 68 \\
\hline Disparos & 67 & 74 & 89 & 77 \\
\hline Pessoas não atingidas no local & 0 & 0 & 0 & 0 \\
\hline Saída & $\begin{array}{c}\text { Evacuação } \\
\text { completa }\end{array}$ & $\begin{array}{c}\text { Evacuação } \\
\text { completa }\end{array}$ & $\begin{array}{c}\text { Evacuação } \\
\text { completa }\end{array}$ & \\
\hline
\end{tabular}




\begin{tabular}{|l|c|c|c|c|}
\hline \multicolumn{5}{|c|}{ Simulação 4 } \\
\hline \multicolumn{1}{|c|}{ Parâmetros de análise } & Teste 1 & Teste 2 & Teste 3 & Média \\
\hline Pessoas no local & 161 & 310 & 347 & 273 \\
\hline Pessoas no local (\%) & 24,62 & 47,54 & 53,23 & 41,80 \\
\hline Vítimas & 160 & 179 & 189 & 176 \\
\hline Disparos & 242 & 250 & 250 & 247 \\
\hline Pessoas não atingidas no local & 0 & 130 & 157 & 96 \\
\hline Saída & $\begin{array}{c}\text { Evacuação } \\
\text { completa }\end{array}$ & $\begin{array}{c}\text { Atirador } \\
\text { neutralizado }\end{array}$ & $\begin{array}{c}\text { Atirador } \\
\text { neutralizado }\end{array}$ & \\
\hline
\end{tabular}

A partir da Tabela 2, pode-se inferir que o número de vítimas de fuzil continua sendo maior do que de pistola, no entanto essa diferença aumentou para em média 108 vítimas a mais na simulação realizada com o fuzil. Com isso, observa-se que, mesmo aumentando a dimensão da saída, a quantidade de munição e o número de pessoas no local tem grande impacto no número de mortes.

Na quinta simulação, os parâmetros de entrada foram população inicial de $\mathbf{1 . 0 0 0}$ pessoas, dimensão da porta igual a $\mathbf{5}$ e a arma utilizada foi a pistola com munição igual a 500. Na sexta e última simulação, os parâmetros foram os mesmos, porém a arma utilizada foi o fuzil. Os resultados das duas últimas simulações são apresentados na Tabela 3.

Tabela 3. Resultados das Simulações 5 e 6

\begin{tabular}{|l|c|c|c|c|}
\hline \multicolumn{5}{|c|}{ Simulação 5 } \\
\hline \multicolumn{1}{|c|}{ Parâmetros de análise } & Teste 1 & Teste 2 & Teste 3 & Média \\
\hline Pessoas no local & 113 & 105 & 111 & 110 \\
\hline Pessoas no local (\%) & 11,2 & 10,4 & 11 & 10,87 \\
\hline Vítimas & 112 & 104 & 110 & 109 \\
\hline Disparos & 135 & 124 & 134 & 131 \\
\hline Pessoas não atingidas no local & 0 & 0 & 0 & 0 \\
\hline Saída & $\begin{array}{c}\text { Evacuação } \\
\text { completa }\end{array}$ & $\begin{array}{c}\text { Evacuação } \\
\text { completa }\end{array}$ & $\begin{array}{c}\text { Evacuação } \\
\text { completa }\end{array}$ & \\
\hline \multicolumn{1}{|c|}{ Simulação 6 } \\
\hline Parâmetros de análise & Teste 1 & Teste 2 & Teste 3 & Média \\
\hline Pessoas no local & 301 & 319 & 306 & 309 \\
\hline Pessoas no local (\%) & 30 & 31,8 & 30,2 & 30,67 \\
\hline Vítimas & 293 & 306 & 302 & 300 \\
\hline Disparos & 500 & 500 & 495 & 498 \\
\hline Pessoas não atingidas no local & 7 & 12 & 0 & 6 \\
\hline Saída & $\begin{array}{c}\text { Atirador } \\
\text { neutralizado }\end{array}$ & $\begin{array}{c}\text { Atirador } \\
\text { neutralizado }\end{array}$ & $\begin{array}{c}\text { Evacuação } \\
\text { completa }\end{array}$ \\
\hline
\end{tabular}

Observa- se, a partir da Tabela 3, que aumentando ainda mais a população e a munição, ainda que se aumente a dimensão da porta para o máximo, o número de vítimas de fuzil foi em média 192 pessoas a mais do que no evento com pistola. Analisando-se os 
dados, podemos concluir que quanto menor a saída e a quantidade de munição, menor será a diferença entre as duas armas, devido ao fato das pessoas demorarem mais para evacuar o ambiente, possibilitando que a maior parte dos projéteis sejam disparados. Devido ao efeito funil, a taxa de acerto dos disparos em direção a saída é alta, como pode ser observado na Figura 2, que apresenta uma simulação gerada com os seguintes parâmetros: população inicial de 711 indivíduos, dimensão da porta em 3, fuzil e atirador portando 500 munições.

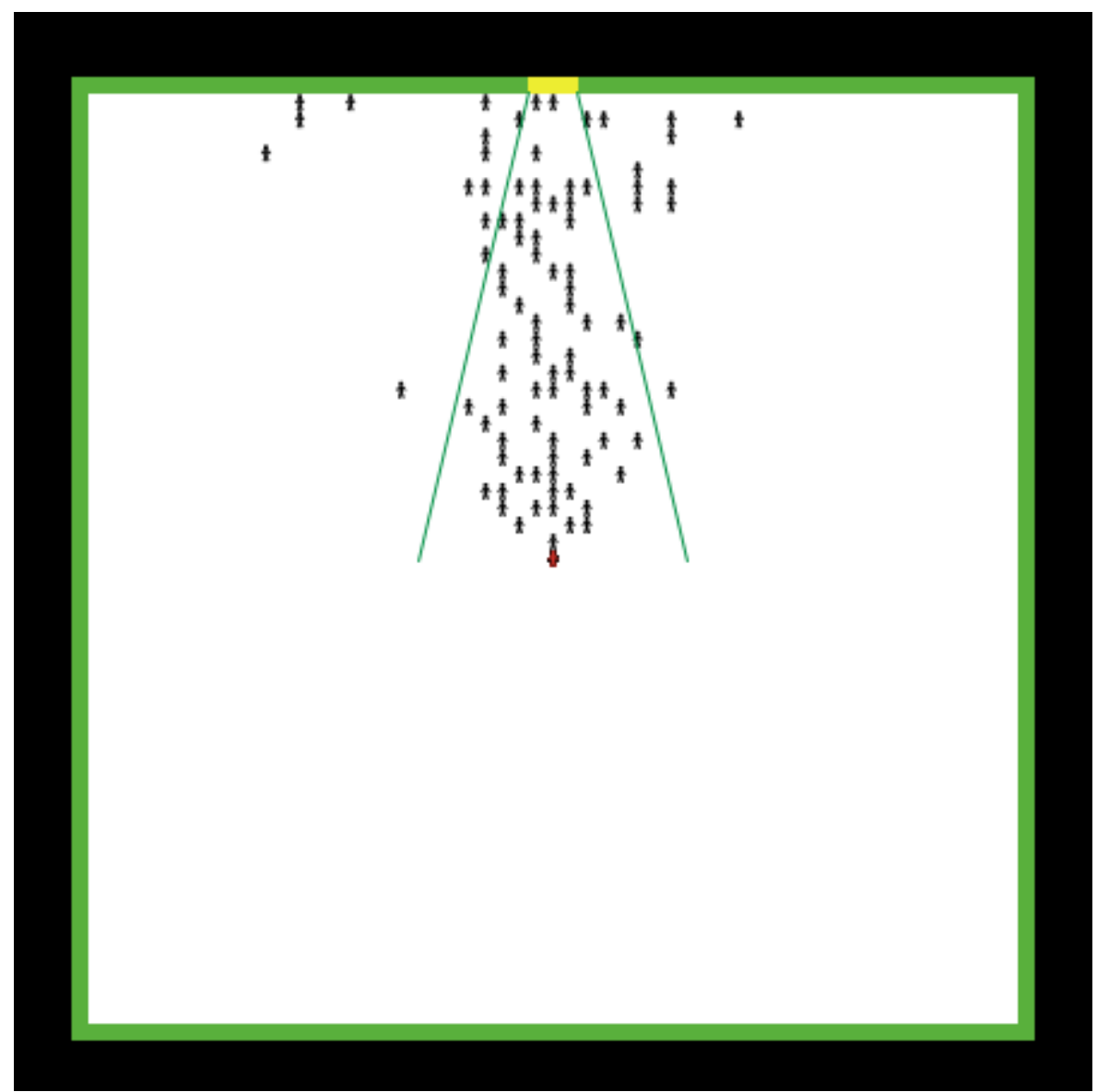

Figura 2. Demonstração do efeito funil, onde ao final da simulação, restaram as pessoas atingidas.

\section{Conclusões Finais}

Através do modelo baseado em agentes proposto foi possível simular eventos de tiroteios em massa em ambientes fechados, utilizando-se dois tipos de armas de fogo: fuzil e pistola. Considerou-se como ambiente fechado o cenário delimitado por paredes e com uma porta de saída, através da qual os agentes que estão na parte interna podem evadir para a parte externa. A simulação permitiu ainda realizar a análise da influência da dimensão da porta de saída, do número de munições que o atirador detém e o número de pessoas no local no número de vítimas.

Das análises realizadas, constatou-se que o fuzil é mais eficiente do que a pistola, sendo que o número de vítimas de fuzil foi superior em todas as simulações. Ou seja, o tipo de arma utilizada tem grande impacto no número de mortes. Além disso, também observou-se que a diferença no número de vítimas entre as duas armas diminui à medida 
em que se aumenta a quantidade de munição e diminui-se a dimensão da porta de saída. Isso deve-se ao efeito funil que se forma, com a concentração de pessoas na área de evacuação.

Considera-se que a modelagem baseada em agentes realizada pode ser utilizada como ferramenta para apoiar a análise de políticas públicas, à medida que permite identificar os parâmetros que minimizam ou potencializam o número de mortes em tiroteios em massa. Neste sentido, políticas pró-armamento poderiam contemplar apenas as armas que apresentam menor eficácia no número de vítimas. A simulação pode ser utilizada ainda na prescrição de diretrizes de segurança, como, por exemplo, na definição de exigências sobre dimensões e número de portas de saída para estabelecimentos com capacidade para determinado número de pessoas.

De acordo com os resultados obtidos na simulação, são desaconselhadas políticas que permitam a posse de armas de maior taxa de disparo, como o fuzil, devido ao seu potencial no número de vítimas. Sugere-se também que seja estabelecido um número máximo de munições permitidas por arma, tendo em vista que a simulação demonstrou que o número de munição impacta positivamente no número de mortes.

Para estudos futuros, sugere-se realizar a análise de outros fatores, como o número de seguranças armados no local e a chegada de policiais após decorrido em um determinado tempo da simulação, o que poderia findar a ação do atirador antes da munição acabar.

\section{Referências}

Briggs, T.W.; Kennedy, W.G. (2016). Active shooter: an agent-based model of unarmed Winter Simulation

Conference, https://doi.org/10.1109/WSC.2016.7822381 [online: accessed 25-may-2020].

Chapman, S.; Alpers, P.; Agho, K.; Jones, M. (2006). Australia's 1996 gun law reforms: faster falls in firearm deaths, firearm suicides, and a decade without mass shootings. Injury Prevention, 12:365-372.

Ferro, W.C.; Teixeira, E.C. (2019). Efeito do Estatuto do Desarmamento sobre as mortes por armas de fogo no Brasil. Revista de Desenvolvimento Econômico, 3(44):56-87.

Folha de São Paulo. (2019). Em metade dos ataques contra escolas no país, armas vieram das casas dos atiradores. https://www1.folha.uol.com.br/cotidiano/2019/03/emmetade-dos-ataques-contra-escolas-no-pais-armas-vieram-das-casas-dosatiradores.shtml [online: accessed 30-may-2020].

Hayes, R.; Hayes, R. (2014). Agent-Based Simulation of Mass Shootings: Determining How to Limit the Scale of a Tragedy. Journal of Artificial Societies and Social Simulation, 17 (2) 5 http://jasss.soc.surrey.ac.uk/17/2/5.html [online: accessed 06may-2020].

Lemieux, F. (2014). Effect of Gun Culture and Firearm Laws on Gun Violence and Mass Shootings in the United States: A Multi-Level Quantitative Analysis. International Journal of Criminal Justice Sciences, 9(1):74-93.

Luca, M.; Malhotra, D.; Poliquin, C. (2020). The impact of mass shooting on gun policy. Journal of Public Economics, 181:1-20. 
McPhedran, S.; Maker, J. (2011). Mass shootings in Australia and New Zealand: a descriptive study of incidence. Justice Policy Journal, 8(1): https://ssrn.com/abstract=2122854 [online: accessed 11-june-2020].

ONU. (2018). Securing our common future: an agenda for disarmament. Office for disarmament affairs, New York, USA.

Xi, J.Y.; Chan, W.K. (2019). Simulation of knife attack and gun attack on university campus using Agent-Based Model and Gis. Winter Simulation Conference, National Harbor, MD, USA, 263-272, doi: 10.1109/WSC40007.2019.9004873. 